\title{
Estimation des populations de Plasmodiophora brassicae Wor. dans les sols
}

Francis ROUXEL \& Maryvonne CADIOU

I.N.R.A., Station de Pathologie végétale, Centre de Recherches de Rennes, B.P. 29, F35650 Le Rheu

RÉSUMÉ

\begin{abstract}
Une méthode originale de dénombrement de l'inoculum de Plasmodiophora brassicae dans les sols est proposée ; son principe est basé sur le piégeage du parasite à l'aide d'une plante très sensible placée dans les conditions optimales pour la manifestation de la hernie : des plantules de chou-chinois sont cultivées en chambre climatisée pendant 6 semaines, soit dans un sol de référence infesté à l'aide de doses croissantes d'inoculum de $P$. brassicae soit dans un mélange constitué de différentes proportions du même sol de référence non infesté et du sol à analyser. En fin de culture, la densité d'inoculum de $P$. brassicae $\left(\mathrm{x}_{1}\right)$ et la proportion de sol à analyser $\left(\mathrm{x}_{2}\right)$ à introduire dans le sol de référence pour provoquer un indice pathologique de $50 \mathrm{p} .100$ sont estimées. Le rapport $\mathrm{x}_{1} / \mathrm{x}_{2} \times 100$ permet ensuite de déterminer la densité d'inoculum par gramme du sol à analyser. Les résultats obtenus sur des sols naturellement infestés sont discutés, et les perspectives d'utilisation de cette méthode envisagées.
\end{abstract}

Mots clés additionnels : Crucifères, hernie, densité d'inoculum, indice pathologique.

\section{SUMMARY}

\begin{abstract}
Assessment of Plasmodiophora brassicae Wor. populations in soil.
An original method to assess Plasmodiophora brassicae populations in soil is described ; the principle consists of detecting the pathogen with very susceptible plants grown in conditions optimal for clubroot development. Chinese cabbage seedlings were grown for 6 weeks, either in a standard soil infested with increasing levels of $P$. brassicae resting spores, or in a mixture of different proportions of non-infested standard soil with the soil for analysis. After cropping, the $P$. brassicae resting spore density $\left(\mathrm{x}_{1}\right)$ and the proportion of soil for analysis $\left(\mathrm{x}_{2}\right)$ in the standard soil producing a disease index of $50 \%$ was estimated. The inoculum density per gram of soil for analysis could be determined by the ratio $x_{1} / x_{2} \times 100$. The data obtained from naturally infested soils are discussed and prospects of using this method are considered.
\end{abstract}

Additional key words : Crucifers, clubroot, inoculum density, disease index.

\section{INTRODUCTION}

La Hernie des Crucifères, due à Plasmodiophora brassicae Wor., constitue le problème parasitaire majeur sur cultures de choux-fleurs; en effet, les méthodes classiques de lutte n'ont pas permis jusqu'ici d'apporter une solution satisfaisante à cette maladie (ROUXEL \& JOUAN, 1982). Ceci s'explique en partie par une connaissance imparfaite de la biologie et de l'écologie du parasite dans les sols : si de nombreux travaux, réalisés surtout à l'étranger, ont montré le rôle du sol $(\mathrm{pH}$, humidité, ...) sur le développement de la maladie (DoBSON et al., 1983; ThUMA et al., 1983 ; CAMPBEll et al., 1985), l'importance de ce facteur sur la conservation du parasite est moins bien connue, en raison des difficultés rencontrées pour dénombrer les populations de $P$. brassicae dans les sols.
Depuis une vingtaine d'années, quelques tentatives de détection et d'estimation des populations de ce parasite obligatoire ont été réalisées: en 1978, BUCZACKI \& OCKENDON ont proposé une méthode d'extraction et d'observation directe des spores de conservation à partir du sol, méthode reprise et modifiée par NAIKI \& KITAZAWA en 1984. Jusqu'ici cependant, aucune de ces méthodes ne donne entière satisfaction et n'a fait l'objet d'applications à l'étude du P. brassicae dans le sol. Parallèlement, certains chercheurs ont essayé d'adapter les techniques de piégeage à l'aide de plantes hôtes sensibles au dénombrement du parasite; ainsi, DATNOFF et al. (1984) réussissent à estimer les populations de P. brassicae dans le cas particulier d'eaux d'irrigation. 
Nous nous sommes inspirés de ces derniers travaux pour tenter de mettre au point une méthode de détection et d'estimation du niveau d'inoculum du P. brassicae dans les sols. Après avoir décrit le principe de la méthode, nous présenterons les résultats de son application au dénombrement de l'inoculum du parasite dans 2 sols maraîchers naturellement infestés.

\section{MATÉRIEL ET MÉTHODES}

\section{A. Technique utilisée}

Le principe consiste à diluer le sol à analyser dans un sol de référence dont on estime préalablement la réceptivité après infestation à l'aide de doses connues d'inoculum de $P$. brassicae, selon la méthode décrite par RouXel \& Regnault (1985). Cette infestation artificielle permet d'établir une corrélation entre les niveaux d'attaques sur une culture sensible et les densités d'inoculum du parasite apportées dans le sol.

Un sol de référence, très sensible à la hernie, est désinfecté 2 fois à la chaleur $\left(110^{\circ} \mathrm{C}-60 \mathrm{~min}\right)$ à $24 \mathrm{~h}$ d'intervalle. Cinq jours plus tard, une partie de ce sol est infestée à l'aide de doses croissantes d'un inoculum de $P$. brassicae obtenu à partir de galles de hernie formées sur racines de chou-fleur. La suspension de spores, obtenue après broyage des galles dans de l'eau, est dénombrée à l'hématimètre. A partir de la suspensionmère, une série de dilutions est effectuée de manière à obtenir, après mélange homogène avec l'ensemble du sol, les concentrations finales de $0,10^{2}, 10^{3}, 10^{4}, 10^{5}$, $10^{6}, 10^{7}$ spores par gramme de sol. La seconde partie du sol de référence est mélangée au sol à analyser de manière à obtenir les proportions suivantes de ce dernier: $0,1,5,10,25,50$ et 100 p. 100 . Chaque traitement est ensuite réparti dans 5 pots de $250 \mathrm{ml}$. Dans chaque pot, 5 graines de chou-chinois, Brassica campestris pekinensis, var. " granaat ", plante très sensible à la hernie, sont semées. Après 6 semaines de culture en chambre climatisée (température de 18 à $20^{\circ} \mathrm{C}$, forte humidité du sol), les plantes sont arrachées et réparties en 4 classes en fonction de la gravité observée de la maladie. Des coefficients (0-0,25-0,5-1) respectivement affectés aux 4 classes permettent le calcul des indices pathologiques pour chaque traitement selon la formule suivante :

\section{IP $=\frac{\sum \text { (Nbre de plantes par classe }}{\times \text { coef. de la classe })} \times 100$.}

\section{B. Interprétation des résultats}

Les indices pathologiques exprimés pour l'ensemble des traitements permettent de calculer les coefficients de corrélation puis d'établir les droites de régression entre la gravité de la maladie et la densité d'inoculum dars le sol de référence ou la proportion de sol d'analyse dans le mélange.

A partir des équations de ces droites, la densité d'inoculum de $P$. brassicae $\left(\mathrm{x}_{1}\right)$ par gramme de sol de référence ainsi que la proportion $\left(\mathrm{x}_{2}\right)$ de sol à analyser permettant d'obtenir un indice pathologique (y) de
50 p. 100 sont estimées. Le rapport $x_{1} / x_{2} \times 100$ permet ensuite de déterminer le nombre de spores de $P$. brassicae par gramme de sol analysé.

\section{Application de la méthode au dénombrement du $P$. brassicae dans 2 sols naturellement infestés}

Ces sols proviennent de 2 parcelles situées dans la zone légumière du littoral nord de la Bretagne: le premier, prélevé sur la Station expérimentale de Pleumeur-Gautier (22), a un $\mathrm{pH}$ de 6,6. Le second, provenant de la Station I.N.R.A. de Kerdevez (29), a un $\mathrm{pH}$ de 5,7 .

Tous deux ont une texture limono-argileuse.

Le sol de référence est le sol de Pleumeur-Gautier préalablement désinfecté à la chaleur. L'inoculum de $P$. brassicae est obtenu à partir de galles de hernie prélevées sur choux-fleurs de même origine.

\section{RÉSULTATS}

Il apparaît une bonne relation entre la gravité des attaques de hernie sur chou-chinois et les densités d'inoculum de $P$. brassicae introduites dans le sol de référence (fig. 1). La présence de symptômes de maladie

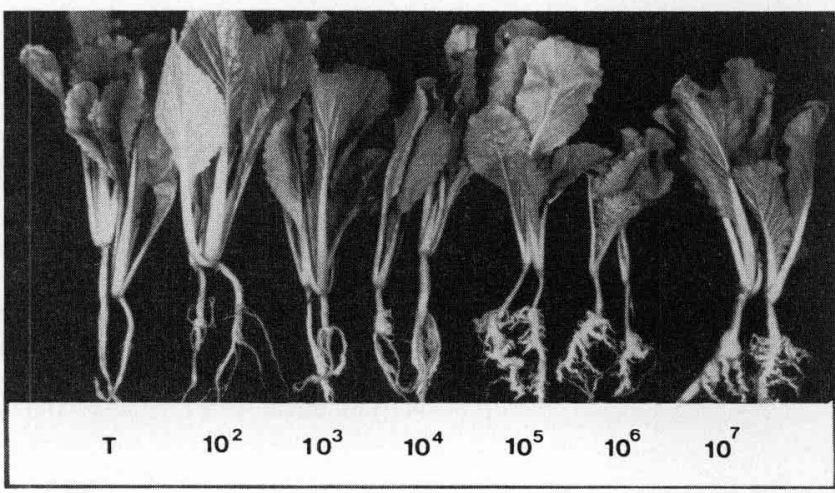

Figure !

Relation entre la gravité des attaques de hernie sur chou-chinois et les densités d'inoculum de $\mathrm{P}$. brassicae introduites dans un sol de référence : 6 concentrations d'inoculum $\left(10^{2}\right.$ à $10^{7}$ spores dormantes $/ g$ de sol $)$ et un témoin non infesté $(T)$.

Relation between clubroot incidence on Chinese cabbage and the inoculum level of $\mathbf{P}$. brassicae introduced into a standard soil : 6 inoculum concentrations ( $10^{2}$ to $10^{7}$ resting spores/g of soil) and a control (T)

dès la concentration de $10^{2}$ spores/g de sol et leur gravité à $10^{5}$ spores/g de sol confirment le niveau élevé de réceptivité de ce sol.

La figure 2 montre que, globalement, la gravité des attaques est plus importante dans les mélanges contenant le sol de Kerdevez que dans ceux réalisés avec le sol de Pleumeur-Gautier. Dans le premier cas, les attaques de hernie apparaissent dès la plus faible proportion ( $1 \mathrm{p} .100)$ et deviennent très importantes à partir de 5 p. 100 du sol de Kerdevez dans le mélange. Dans le second cas, la maladie apparaît à la dilution de 


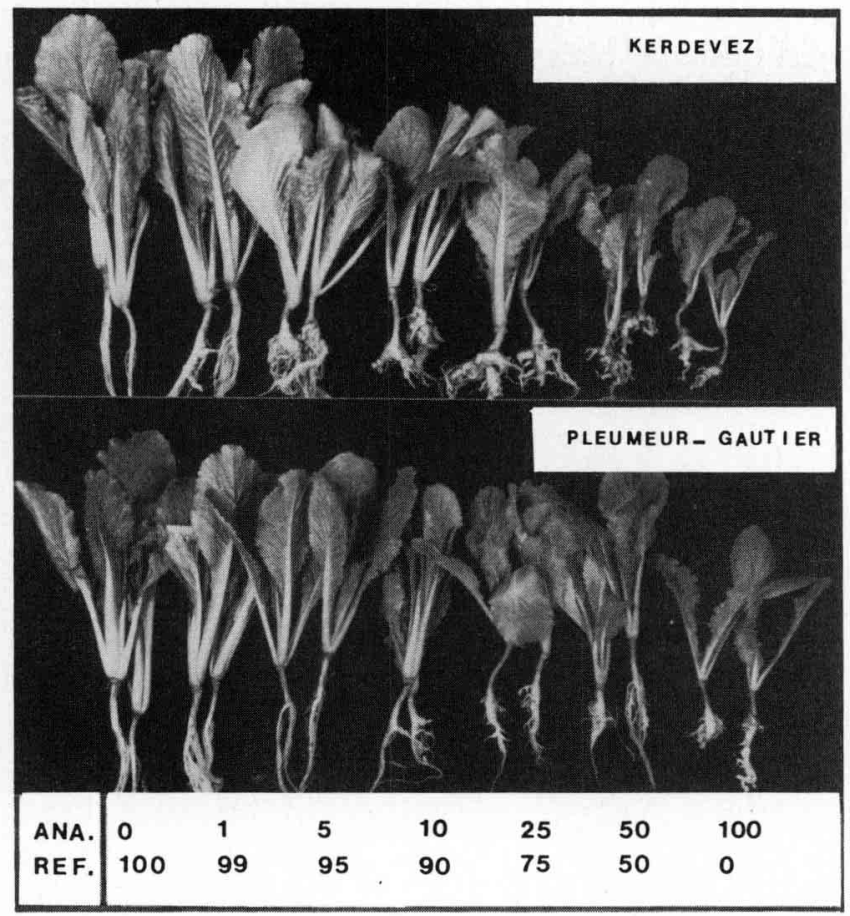

Figure 2

Relation entre la gravite des attaques de hernie sur chou chinois et les différentes proportions des 2 sols à analyser (ANA) dans le sol de référence $(R E F)$ : 7 pourcentages $(0$ à $(00)$ de chaque sol à analyser (KERDEVEZ \& PLEUMEUR-GAUTIER).

Relation between clubroot incidence on Chinese cabhage and the proportion of two analyzed soils (ANA) in the standard soil (REF): 7 percentages (0) to 100) for each analyzed soil (KERDEVEZ \& PLEUMEURGAUTIER).

10 p. 100 ; elle n'est très grave que lorsque le sol de Pleumeur-Gautier est pur (100 p. 100).

Les équations des droites de régression $(r=0,96)$ entre les indices pathologiques mesurés et les dilutions de chacun des 2 sols à analyser dans le sol de référence (fig. 3) permettent de définir la proportion de chaque sol provoquant un indice pathologique de $50 \mathrm{p} .100$ dans le mélange: 5.2 p. $100(\log \mathrm{D}=0.72)$ pour le sol de Kerdevez; 23,6 p. $100(\log \mathrm{D}=1,37)$ pour le sol de Pleumeur-Gautier.

La figure 4 montre que, pour le sol de référence, ce même indice pathologique de 50 p. 100 est obtenu pour une concentration d'inoculum de $5.10^{3}$ spores/g de sol $(\log C=3,73)$.

Les densités d'inoculum dans les 2 sols peuvent donc être estimées à :

$\frac{5.10^{3}}{5,2} \times 100$ soit $10^{5}$ spores de P. brassicae/gramme

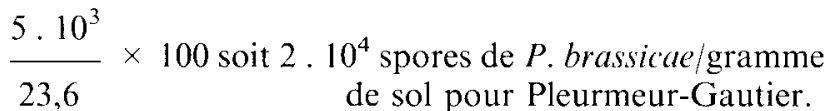

\section{CONCLUSION-DISCUSSION}

La méthode présentée ici, basée sur le piégeage par une plante sensible, le chou-chinois, permet d'estimer les populations de $P$. brassicae dans des sols naturellement

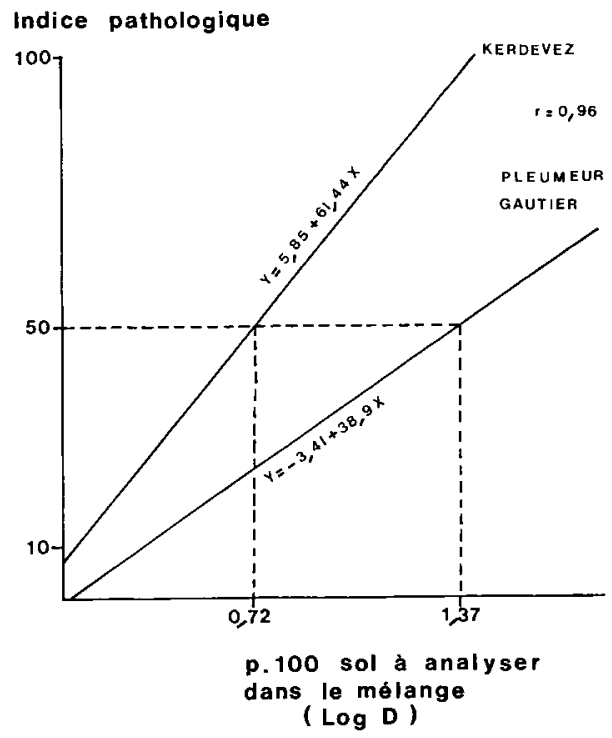

Figure 3

Régressions linéaires entre la gravité des attaques de hernie (IP) sur chou chinois et les pourcentages de chaque sol à analyser ( Log D) dans le sol de référence. Proportions de sol permettant d'obtenir un indice pathologique de 50 p. $100:$ KERDEVEZ $=5,2$ p. $100 ;$ PLEUMEURG.ALTIER $=23,6$ p. 100 .

Linear regressions between clubroot incidence $(I P)$ on Chinese cabbage and the percentage of each analyzed soil (Log D) in the standard soil. The proportion of soil to obtain a $50 \%$ disease index was: KERDE$V E L=5.2 \% ;$ PLEUMEUR-GAUTIER $=23.6 \%$.

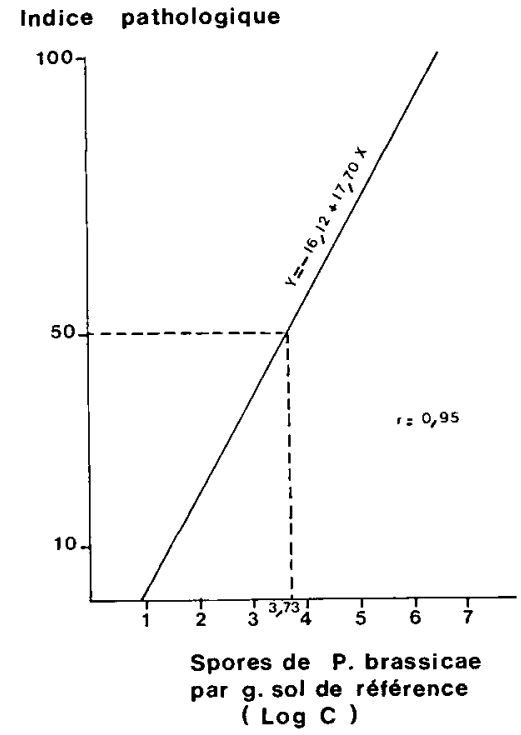

Figure 4

Régression linéaire entre la gravité des attaques de hernie (IP) sur chou chinois et les densités d'inoculum de P. brassicae $(\log C)$ introduites dans lo sol de référence. Densité d'inoculum permettant d'obtenir un indice pathologique de 50 p. $100=5 \cdot 10^{3}$ spores de repos/g de sol.

Linear regression betucen clubroot incidence $(I P)$ on Chinese cabhage and the inoculum levels of $\mathrm{P}$. brassicae (Log C) introduced into the standard soil. The inoculum level to ohtain a $50 \%$ disease index was. $5 \cdot 10^{3}$ resting spores/g of soil.

infestés. Elle constitue une approche originale puisque jusqu'ici le piégeage a surtout été utilisé pour estimer le potentiel infectieux (Colhoun, 1957; Melville \& HAWKEN, 1967) et non la densité d'inoculum des sols. 
L'utilisation d'un seul sol de référence permet une standardisation de la méthode par rapport à celle proposée par DATNOFF et al. (1984) et facilite son application. L'intérêt de cette approche est d'autant plus grand que les différentes méthodes d'extraction et d'observation directe (BUCZACKI \& OCKENDON, 1978 ; NAIKI \& KITAZAWA, 1984), que nous avons essayées par ailleurs (non publié), se sont avérées mal adaptées à des sols limono-argileux. Cette première application du piégeage au dénombrement de l'inoculum du $P$. brassicae dans les sols laisse entrevoir de nombreuses perspectives d'utilisation :

- étude des niveaux de contamination des sols de culture,

- étude du comportement du $P$. brassicae dans les sols en fonction de leurs niveaux de réceptivité,

- évolution des populations du parasite dans les sols après traitements fongicides.

Certaines limites d'utilisation de la méthode ont cependant été mises en évidence; elles concernent notamment les exigences en temps (test de 6 semaines), mais aussi le biais éventuellement introduit par des caractéristiques physico-chimiques ou microbiologiques particulières du sol analysé qui peuvent modifier la réceptivité du mélange par rapport à celle du sol de référence. Enfin, le piégeage reste avant tout une méthode indirecte de dénombrement; il met en évidence un inoculum réellement infectieux, mais risque de sousestimer la densité d'inoculum du sol (spores dormantes par exemple). Certaines améliorations et vérifications restent donc à réaliser pour mieux connaître les contraintes de la méthode, mais aussi ses potentialités.

En pratique, on peut déjà penser qu'il serait intéressant de coupler dénombrement des populations de $P$. brassicae par piégeage et dénombrement par observation directe, les 2 approches étant tout à fait complémentaires.

\section{RÉFÉRENCES BIBLIOGRAPHIQUES}

Buczacki S. T., Ockendon J. G., 1978. A method for the extraction and enumeration of resting spores of Plasmodiophora brassicae from infested soil. Ann. appl. Biol., 88, 363-367.

Campbell R. N., Greathead A. S., Myers D. F., De Boer G. J., 1985 Factors related to control of clubroot of crucifers in the Salinas Valley of California. Phytopathology, 75 : 665-670.

Colhoun J., 1957. A technique for examining soil for the presence of Plasmodiophora brassicae Wor. Ann. appl. Biol., 45, 559-565.

Datnoff L. E., Lacy G. H., Fox J. A., 1984. Occurrence and populations of Plasmodiophora brassicae in sediments of irrigation water sources. Plant Dis., 68, 200-203.

Dobson R. L., Gabrielson R. L., Baker A. S., Bennett L., 1983. Effects of lime particle size and distribution and fertilizer formulation on clubroot disease caused by Plasmodiophora brassicae. Plant Dis., 67, $50-52$.
Melville S. C., Hawken R. H., 1967. Soil testing for clubroot in Devon and Cornwall. Plant Pathol., 16, 145-147.

Naiki T., Kitazawa S., 1984. On a method for direct assessment of resting spores of Plasmodiophora brassicae in soil. Proc. kansai Pl. Prot. Soc, $26: 9-14$

Rouxel F., Jouan B., 1982. La Hernie des Crucifères in " Les Maladies des Plantes ". ACTA, Paris, 74-84.

Rouxel F., Regnault Y., 1985. Comparaison de la réceptivité des sols à la Hernie des Crucifères: Application à l'évaluation des risques sur quelques sols à culture de Colza oléagineux. lères J., études maladies des plantes. A.N.P.P., 2, 375-383.

Thuma B. A., Rowe R. C., Madden L. V., 1983. Relationships of soil temperature and moisture to clubroot (Plasmodiophora brassicae) severity on radish in organic soil. Plant Dis., 67, 758-762. 